\title{
Dissecting Local Atomic and Intermolecular Interactions of Transition-metal Ions in Solution with Selective X-ray
}

\section{Spectroscopy}

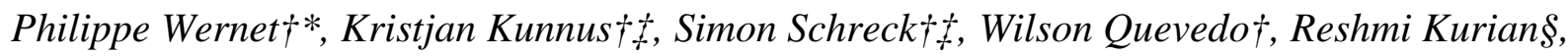
Simone Techert\#, Frank M. F. de Groot\$, Michael Odeliusl, Alexander Föhlisch †t

† Institute für Methods and Instrumentation for Synchrotron Radiation Research, HelmholtzZentrum Berlin für Helmholtz-Zentrum Berlin für Materialien und Energie GmbH, AlbertEinstein-Str. 15, 12489 Berlin, Germany

‡ Institut für Physik und Astronomie, Universität Potsdam Karl-Liebknecht-Strasse 24/25 14476 Potsdam, Germany

$\S$ Inorganic Chemistry \& Catalysis, Debye Institute for Nanomaterials Science, Utrecht University, Universiteitsweg 99, 3584 CG, The Netherlands

\# Structural Dynamics of (Bio)chemical Systems, Max Planck Institute for Biophysical Chemistry, 37070 Göttingen, Germany

\| Department of Physics, Stockholm University, AlbaNova University Center, 10691 Stockholm, Sweden 


\begin{abstract}
Determining covalent and charge-transfer contributions to bonding in solution has remained an experimental challenge. Here the quenching of fluorescence-decay channels as expressed in dips in the L-edge x-ray spectra of solvated 3d transition-metal ions and complexes was reported as a probe. With a full set of experimental and theoretical ab initio L-edge x-ray spectra of aqueous $\mathrm{Cr}^{3+}$ including resonant inelastic $\mathrm{x}$-ray scattering we address covalency and charge-transfer for this prototypical transition-metal ion in solution. We dissect local atomic effects from intermolecular interactions and quantify x-ray optical effects. We find no evidence for the asserted ultrafast charge-transfer to the solvent and show that the dips are readily explained by xray optical effects and local atomic state dependence of the fluorescence yield. Instead we find, besides ionic interactions, a covalent contribution to the bonding in the aqueous complex of ligand to metal charge transfer character.
\end{abstract}




\section{TOC Graphic:}

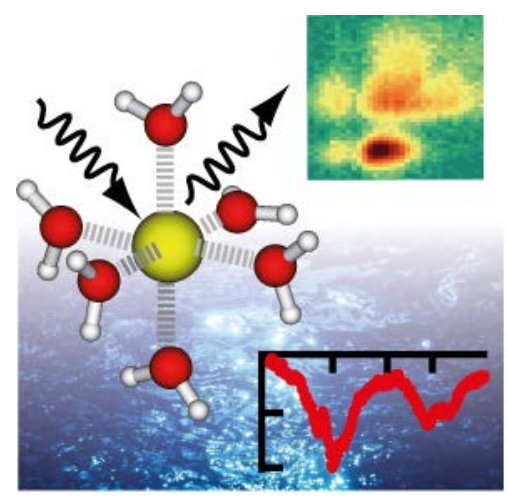

KEYWORDS Charge transfer to solvent, dark channel fluorescence yield, inverse partial fluorescence yield, total fluorescence yield, resonant inelastic x-ray scattering, transition metal ion, aqueous solution. 
The transfer of charge from a solvated ion or molecular complex to the surrounding solvent is an elementary step in numerous reactions in nature and in technological processes. Understanding charge transfer to solvent effects in molecular systems is thus of primary importance and x-ray spectroscopy with its specificity to elemental and chemical sites promises to add a new suite of powerful tools for their investigation (1). Specifically, time-resolved x-ray spectroscopy (2-11) appears to be the most direct way of probing charge transfer initiated by a pump pulse and probed as a function of time by scanning the delay of a short x-ray probe pulse. However, such effects can proceed on the few-femtosecond time scale and it is currently very challenging to achieve a corresponding time-resolution in pump-probe x-ray spectroscopy experiments. Therefore, it has been attempted to make use of the core-hole lifetime of a few femtoseconds to study ultrafast charge transfer processes for various systems such as for adsorbates on surfaces (12-18), ice (19) and aqueous solutions (20). Using resonant soft x-ray spectroscopy a coreelectron is excited into an unoccupied state of the system and the probability for it to transfer to the surroundings during the few-femtosecond core-hole lifetime will influence the core-hole decay channels. The Auger decay signal is thus the stronger, the higher the probability for its transfer to the surrounding medium during the core-hole lifetime is $(15,17,18)$.

As core-excited states can decay via Auger-electron ejection or fluorescence, the question arises whether such ultrafast electron-transfer processes are observable in the fluorescence decay channel as well. This relates to the more general questions as to whether and how bonding and charge-transfer processes can be revealed by with x-ray spectroscopy. Along these lines, Aziz et al. recently reported the so called "dark channel fluorescence yield” method $(21,22)$. They found features in the total fluorescence yield (TFY) spectra when scanning the L-edges of 3d transition 
metal ions and complexes in solution at high concentration that dip below the fluorescence background of the solvent. It was claimed that this could be used as a new tool to study charge transfer to solvent effects (21-24). The dips where attributed, first, to ultrafast electron transfer from the metal d-orbitals to the solvent (21-24) and, second, to a competition of solute and solvent fluorescence (25). Earlier, Näslund et al. proposed strong orbital mixing between the 3d orbitals of Fe ions solvated in water based on an analysis of the $\mathrm{O}$ K-edge absorption spectrum of aqueous solutions (26) without making a connection to any charge transfer. In the context of “dark channel fluorescence yield” Aziz et al. interpreted this orbital mixing as supportive for the assertion of ultrafast electron transfer from the metal d-orbitals to the solvent as deduced from the metal-ion L-edge spectra (21). This electron transfer would occur during the core-hole lifetime of core-excited state in the metal-ion: As the electron excited upon x-ray absorption would have transferred to the solvent, the corresponding fluorescence decay channel would be quenched leaving a dip in the metal-ion L-edge spectrum (21-24). It was claimed that the transfer would be the more efficient and correspondingly the dip would be the deeper, the stronger the orbital overlap between the metal d-orbitals and the solvent orbitals would be. While resonant photoelectron and fluorescence yield spectroscopy of aqueous $\mathrm{Co}^{2+}$ ions have been used to allegedly confirm the origin of "dark channel fluorescence" $(27,28)$ two investigations based on calculated spectra of free metal ions (29) and measured spectra of a solid compound (30) recently proposed alternative explanations for the observed dips.

Here we address the bonding and possible charge transfer via "dark channel fluorescence yield" of ions in aqueous solution. A full set of experimental x-ray data with calculated spectra from an ab initio theory enable us to unambiguously disentangle local atomic effects from intermolecular 
interactions. In addition we accurately quantify all "x-ray optical effects". We address in particular the possible explanation of dips in the TFY metal ion spectra by charge transfer to solvent for the exemplary case of $\mathrm{Cr}^{3+}$ ions solvated in water.

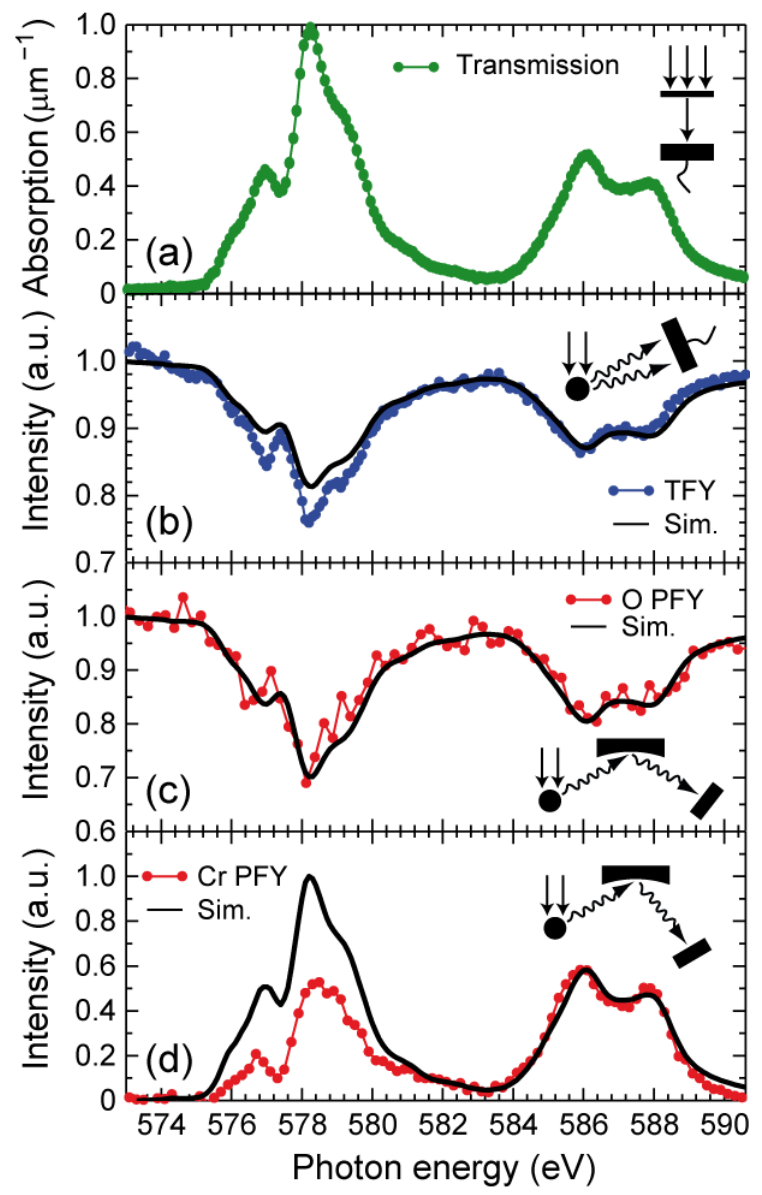

Figure 1. Comparison of experimental and simulated x-ray spectra at the $\mathrm{Cr}_{3}$, 2 -edges with schematical depictions of the corresponding experimental arrangement in the insets. (a) Experimental x-ray absorption spectrum measured in transmission mode. (b) Measured total fluorescence yield (TFY) spectrum (circles) and simulated TFY spectrum (line). (c) Measured partial fluorescence yield (PFY) spectrum by detecting the oxygen K emission (O PFY spectrum, circles) and simulation of the O PFY spectrum, (d) Measured PFY spectrum by detecting the $\mathrm{Cr}_{3,2}$ emission (Cr PFY spectrum, circles) and simulation of the Cr PFY spectrum with constant fluorescence yield of $3.7 \times 10^{-3}$. All spectra are normalized to 1 at maximum. 
The x-ray absorption spectrum of aqueous $\mathrm{Cr}^{3+}$ ions as measured in transmission mode is compared in Figure 1 with the TFY spectrum, the $\mathrm{O}$ and $\mathrm{Cr}$ partial fluorescence yield (PFY) spectra (O and Cr PFY, respectively) and simulated spectra corresponding to the respective detection modes. The $\mathrm{O}$ and $\mathrm{Cr}$ PFY spectra denote $\mathrm{x}$-ray spectra where, as in all other spectra, the incident photon energy was scanned across the $\mathrm{Cr}$ L-edges while the fluorescence from the $\mathrm{O}$ K-edge and Cr L-edges was detected, respectively (see EXPERIMENTAL SECTION). All simulated spectra (denoted with "Sim." in the figures) were calculated with the standard formula for fluorescence detected x-ray absorption $(31,29)$ (see THEORETICAL CALCULATIONS). They include various "x-ray optical effects" such as a variation of the solvent or background fluorescence intensity due to changes in relative solute-solvent absorption strengths when crossing the solute absorption edges and effects due to saturation and self-absorption. The simulations rely on the experimental Cr L- [Figure 1(a)] and O K-edge (not shown) transmission spectra as inputs for solute and solvent absorption cross sections and on experimental Cr L-edge RIXS [Figure 2(a)] and O K-edge x-ray emission spectra (not shown) to account for the spectral distributions of emitted photons. They do not include any effects on the spectra due to a hypothetical charge transfer to the solvent.

While the transmission-mode spectrum in Figure 1(a) reflects the true absorption cross section, the TFY spectrum [Figure 1(b)] can be characterized by dips for all multiplet components at both the $\mathrm{L}_{3}(578 \mathrm{eV})$ and the $\mathrm{L}_{2}$ edges $(587 \mathrm{eV})$. The simulated TFY spectrum in Figure 1(b) reproduces the dips in the measured TFY spectrum except for a small deviation of the intensities at the $\mathrm{L}_{3}$ edge. This demonstrates that the dips in the Cr TFY spectrum are largely due to x-ray optical effects. The measured and simulated O PFY spectra in Figure 1(c) prove in particular that 
they are mainly due to a corresponding variation in the background (solvent $\mathrm{O}$ K-edge) fluorescence. The perfect match between measured and simulated O PFY spectra in Figure 1(c) is furthermore consistent with the finding that it is possible to extract the true absorption spectrum from PFY spectra as recently shown by Achkar et al. (32). The Cr PFY spectrum in Figure 1(d) strongly deviates from the absorption cross section [Figure 1(a)]. In contrast to the transmission mode spectrum, $\mathrm{L}_{3}$ and $\mathrm{L}_{2}$ edges are approximately equally intense in the $\mathrm{Cr}$ PFY spectrum. This is a clear manifestation of state- or incident-photon-energy dependence of the $\mathrm{Cr}$ fluorescence yield. This effect was recently predicted by calculations in Kurian et al. (unpublished results) and earlier by calculations in ref. 33 and it is detailed below with Figure 2. The simulated Cr PFY spectrum in Figure 1 (d) strongly overestimates the intensity at the $\mathrm{L}_{3}$ edge compared to the measured spectrum as the simulation is based on a constant fluorescence yield of $3.7 \times 10^{-3}$ (34) for all states and in particular for both edges. The question arises whether the small disagreement of the simulated TFY with experiment at the $\mathrm{L}_{3}$ edge [Figure 1(b)] is due to this shortcoming of the simulation with constant fluorescence yield or whether other effects such as charge transfer to solvent are needed to explain it.

Before refining the Cr PFY simulation we now turn to a short discussion of the state dependence of the Cr fluorescence yield. This is done with the measured resonant inelastic x-ray scattering (RIXS) plane in Figure 2 (a) where fluorescence intensities at the Cr L-edges are plotted versus the incident photon energy and the energy transfer. In Figure 2 (b) we plot Cr PFY spectra as extracted for the given energy transfer ranges where the spectrum for transfers of -2 to $18 \mathrm{eV}$ corresponds to the aforementioned Cr PFY spectrum and as displayed in Figures 1 and 3. 


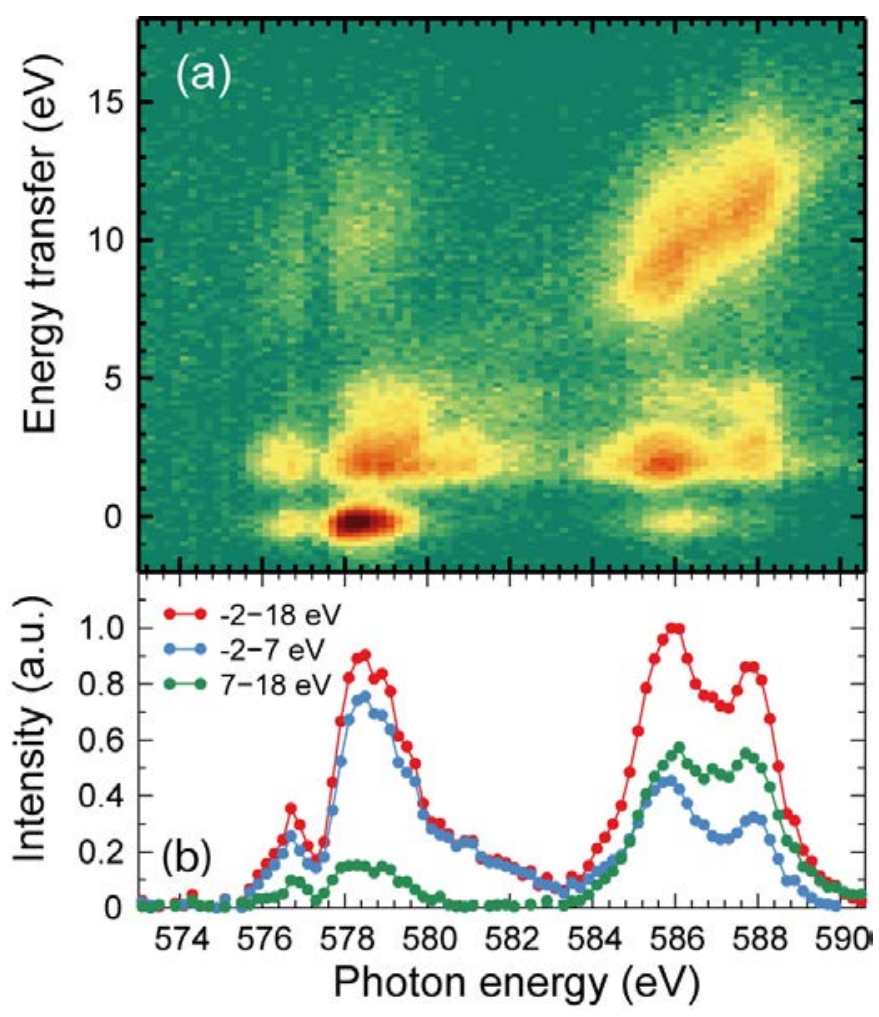

Figure 2. $\mathrm{Cr}_{3,2}$ resonant inelastic x-ray scattering (RIXS) spectra. (a) Full RIXS plane displaying fluorescence intensities in a false-color plot representation with intensities encoded in color (green: small intensity, dark red: large intensity) versus energy transfer (vertical) and incident photon energy (horizontal). (b) Partial fluorescence yield spectra as extracted from (a) for the given energy transfer regions (circles connected by lines). The spectrum for energy transfers of $-2 \mathrm{eV}$ to $18 \mathrm{eV}$ is the same as in Fig. 1(d). It is normalized to 1 at maximum and all other spectra are plotted with respective intensities.

The energy transfer is simply defined as the difference between the incident photon energy and the fluorescence or emission energy. The striking feature in the RIXS plane to be noted first is the strong intensity at the $\mathrm{L}_{2}$ edge and at energy transfers of around $11 \mathrm{eV}$. This is due to CosterKronig (CK) decays (35) of $\mathrm{L}_{2}$ holes as can be easily justified by the approximately $9 \mathrm{eV}$ difference to the energy losses of the main fluorescence centered approximately at $2 \mathrm{eV}$ at the $\mathrm{L}_{2}$ edge and in perfect correspondence with the $\mathrm{L}_{3}-\mathrm{L}_{2}$ spin-orbit splitting of approximately $9 \mathrm{eV}$. 
Such CK signals can be common features in x-ray spectra and have been recently also discussed in $\mathrm{Fe}^{2+}$ ions in aqueous solution (36).

The CK signal observed here does not explain the equal intensity of $L_{3}$ and $L_{2}$ edges in the PFY spectrum as CK decays reshuffle intensities in the RIXS plane along the vertical (energy transfer) axis but not between $\mathrm{L}_{3}$ and $\mathrm{L}_{2}$ edges. Instead, differences of the fluorescence yields for corresponding energy transfer regions at the $\mathrm{L}_{3}$ compared to the $\mathrm{L}_{2}$ edge as apparent from the RIXS plane in Figure 2 (a) are responsible for the deviation of the Cr PFY spectrum [transfers of -2 to $18 \mathrm{eV}$ in Figure 2 (b)] from the absorption spectrum. Note, for example, that the fluorescence intensities at or close to zero energy transfer are stronger at the $\mathrm{L}_{3}$ compared to the $\mathrm{L}_{2}$ edge. Instead, the fluorescence is approximately equally strong at $\mathrm{L}_{3}$ and $\mathrm{L}_{2}$ for transfers of around $2 \mathrm{eV}$ and there is apparent fluorescence intensity at comparably large energy transfers of around $10 \mathrm{eV}$ at the $\mathrm{L}_{3}$ edge. These variations are responsible for the discrepancy between the $\mathrm{Cr}$ PFY and absorption spectra and with the calculations discussed below we demonstrate that they are due to state-dependent variations in the fluorescence decays rates.

In order to refine our simulations by accounting for the variations of the fluorescence yields, we fitted the simulated to the measured Cr PFY spectrum. The best match is displayed in Figure 3 (a) and we find it for fluorescence yields of $2.0 \times 10^{-3}$ for $\mathrm{L}_{3}$ and $3.7 \times 10^{-3}$ for $\mathrm{L}_{2}$. 


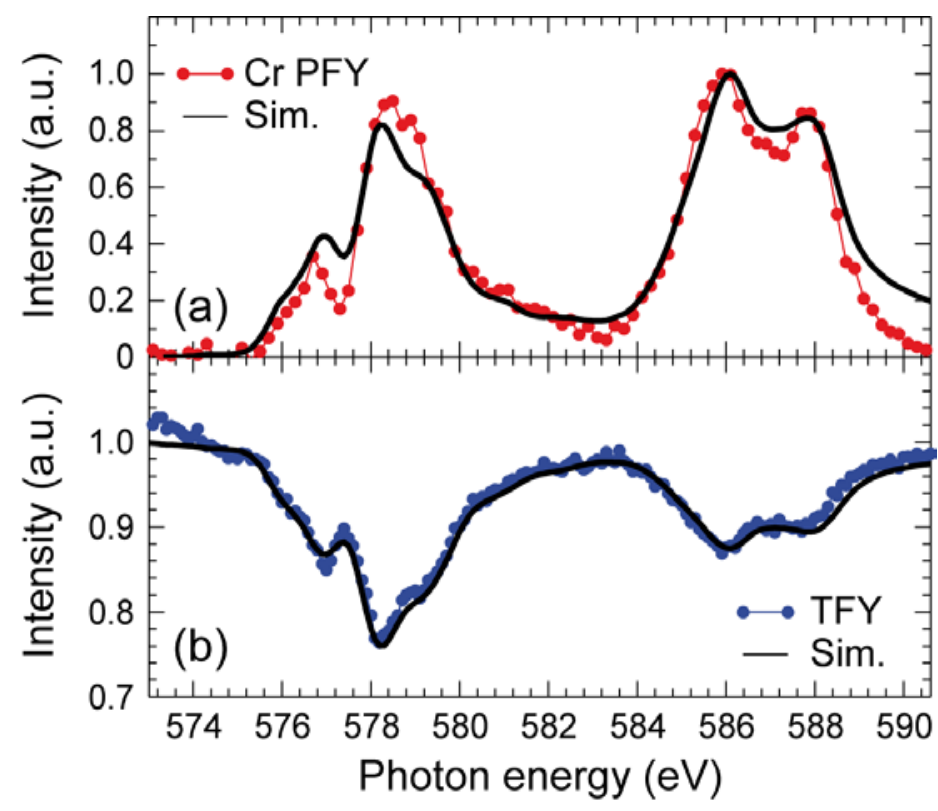

Figure 3. Comparison of experimental and refined simulated x-ray spectra at the $\mathrm{Cr}_{3,2}$-edges. (a) Measured $\mathrm{Cr} \mathrm{L}_{3}$, ${ }_{2}$ PFY spectrum [Cr PFY spectrum, circles, same as Fig. 1(d)] and simulation of the Cr PFY spectrum (line) with different fluorescence yields for $L_{3}\left(2.0 \times 10^{-3}\right)$ and $L_{2}\left(3.7 \times 10^{-3}\right)$ as obtained from a best fit of the simulated to the experimental data. (b) Measured TFY spectrum (circles) and simulated TFY spectrum (line) with the fluorescence yields for $L_{3}$ and $L_{2}$ as determined in (a). All spectra are normalized to 1 at maximum.

With this refinement we find a perfect match between the measured and simulated Cr TFY spectra as shown with the comparison in Figure 3 (b). This demonstrates that the dips in the TFY spectrum are solely due to x-ray optical effects as accounted for by the standard formula for fluorescence yield x-ray absorption spectroscopy and a state- or incident-photon-energy dependence of the fluorescence yield. There is no need to evoke a new process such as "dark channel fluorescence yield” or an additional intermolecular mechanism such as charge transfer to the solvent to explain the dips. With this we fully confirm the theoretical analysis of TFY spectra of free $\mathrm{Fe}^{3+}$ ions by de Groot (29). A more detailed presentation of the simulations of the x-ray optical effects will be published elsewhere. Our simulations indicate that the main contributions to the x-ray optical effects are the variation of the background fluorescence confirming the claim 
in ref. 30 and a variation of the experimental geometry which, in turn, mainly affects the contribution of saturation (31). Together with the state-dependent fluorescence yield as detected here for aqueous $\mathrm{Cr}^{3+}$ we can, in general, explain the dips in the TFY of 3d transition metal ions and complexes in solution. In particular, some approximate trends can be readily explained (29, 30): The strength of the dips in the TFY spectra of 3d transition metal ions in water decreases for increasing Z (21) and with increasing fluorescence yield of the solute compared to the fluorescence yield of $\mathrm{O}$ in water. Furthermore, for a given $3 \mathrm{~d}$ transition metal ion the strength of the dip decreases when going from water to alcohols as solvent due to the increased absorption of the additional $\mathrm{C}$ atoms in the alcohol molecules. Finally, no dips where observed in several solvated species $(37,38)$ as can be explained by their low concentration compared to the concentrations used here and in the studies where dips were reported (21-24, 27, 28).

The questions remain as to what is the origin of the state-dependent fluorescence decay rate in aqueous $\mathrm{Cr}^{3+}$ and whether it can be related to interactions between the metal ion and the solvent molecules in general or to a charge transfer to the solvent in particular. In order to answer these questions we performed two types of calculations that mainly differ in the way the intermolecular interactions are treated. The calculated Cr L-edge x-ray absorption and PFY spectra are displayed in Figure 4. 


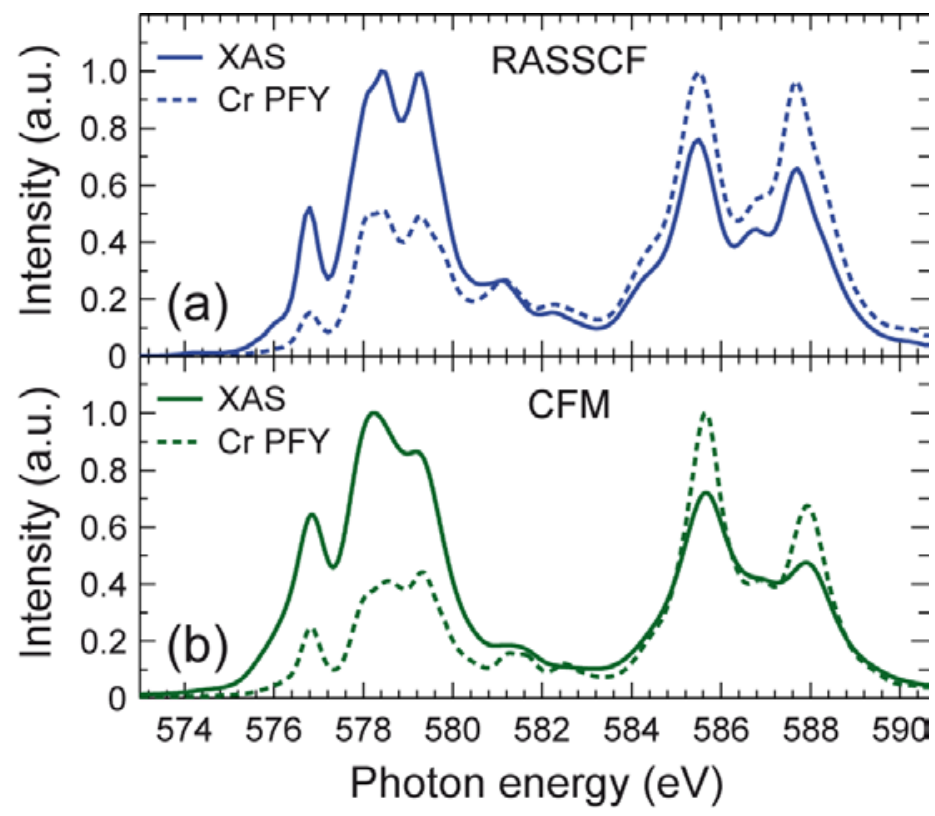

Figure 4. Theoretical x-ray absorption spectra (XAS, solid lines) and Cr partial fluorescence yield (Cr PFY) spectra (dashed lines) as calculated within the RASPT2 (a) and CFM (b) approaches (see text). All spectra are normalized to 1 at maximum.

The ab initio RASPT2 calculations of a $\left[\mathrm{Cr}\left(\mathrm{H}_{2} \mathrm{O}\right)_{6}\right]^{3+}$ complex are based on a molecular-orbital approach including the interactions of the $\mathrm{Cr}^{3+}$ ion with the water ligands with an accurate explicit treatment of both electrostatic and covalent intermolecular interactions. The semiempirical CFM calculations are based on localized atomic orbitals (39) and the ligand environment is approximated by a crystal field using the ligand field parameter 10Dq and by scaling of the Slater integrals without explicit inclusion of intermolecular interactions. The calculated x-ray absorption and PFY spectra in Figure 4 agree well with the corresponding measured spectra in Figure 1. This confirms the well-known fact that L-edge absorption features in the spectra of 3d transition-metal ions are dominated by allowed transitions to the atomic multiplet states of the open-shell $2 \mathrm{p}^{5} 3 \mathrm{~d}^{\mathrm{n}}$ configuration ( $\mathrm{n}=4$ for aqueous $\mathrm{Cr}^{3+}$ ). Note that this is the only core-excited configuration included in our calculations and, in particular, no 
configurations where charge has transferred to the ligands need to be included to reach agreement with experiment. For sure, electrons do not transfer during the core-hole lifetime in aqueous $\mathrm{Cr}^{3+}$. Furthermore, the calculations reproduce the measured deviation of the PFY spectrum compared to the absorption spectrum. In both calculations for the PFY spectra the fluorescence yields were approximated to be proportional to the fluorescence decay rates (see the Supporting Information). This confirms that inclusion of state-to-state variations in the fluorescence decay rates is sufficient to explain the difference between the PFY and absorption spectra.

It is important not to confuse charge transfer processes as discussed here with covalent interactions which are also often described in terms of charge transfer. Our RASPT2 calculations can be used to infer details of the intermolecular interactions in the $\left[\mathrm{Cr}\left(\mathrm{H}_{2} \mathrm{O}\right)_{6}\right]^{3+}$ complex. They show that covalent interactions between $\mathrm{Cr}^{3+}$ and $\mathrm{H}_{2} \mathrm{O}$ are definitely present. However, the mixing of $\mathrm{Cr} 3 \mathrm{~d}$ orbitals with the water lone pair $1 \mathrm{~b}_{2}$ and the $3 \mathrm{a}_{1}$ orbitals is weak and bonding is mostly ionic. As the Cr 3d orbitals are only partially occupied the weak covalent interaction is of ligand to metal charge transfer type resulting in a small increase of 3d-orbital occupancy. Charge is thus partially transferred in terms of orbital mixing in the opposite direction compared to what had been evoked by Aziz et al. (21). In a ligated metal ion, charge transfer in the framework of covalent bonding is always referenced to the metal ion without ligands. This is different from the transient charge-transfer process in core-excited states as postulated by Aziz et al..

Finally, both our theoretical approaches correctly describe the essential local atomic electron repulsion and spin-orbit interactions in the core-excited states. A large number of states are 
present as a result of the many possible determinants of the $2 \mathrm{p}^{5} 3 \mathrm{~d}^{4}$ configuration in $\mathrm{Cr}^{3+}$. These determinants are mixed due to the inter- and intra-atomic interactions, including spin-orbit coupling. It is thus no surprise that the core-excited states which are composed of determinants with different symmetry and spin can overlap differently with the valence-excited RIXS final states. The state dependent fluorescence rate is thus a local atomic effect (29) and simply results from the large number of states of the $\mathrm{Cr}^{3+} 2 \mathrm{p}^{5} 3 \mathrm{~d}^{4}$ configuration. This is of utmost importance as it shows that one cannot infer from a variation of the fluorescence yield a delocalization of the excited electron or even on orbital mixing or hybridization as recently insinuated (27, 28, 40, 41).

In conclusion we present a consistent set of experimental and theoretical data for a transitionmetal ion in solution that allows us to disentangle local atomic effects, intermolecular interactions and x-ray optical effects. We show in particular that the dips in the Cr L-edge TFY spectrum of aqueous $\mathrm{Cr}^{3+}$ cannot be interpreted in terms of charge transfer to solvent effects. We find no evidence for the asserted "dark channel fluorescence". Instead, the TFY spectra are dominated by straight-forward x-ray optical and local atomic effects. Mainly, these are a variation of the solvent or background fluorescence intensity and of saturation. In addition, we find a strong state- or incident-photon energy dependence of the fluorescence yield due to a state dependence of the fluorescence decay rates. This local atomic effect in turn is a natural consequence of the large number of core-excited states at the Cr L-edges as a result of the partially filled 3d shell. We address the bonding in the $\left[\mathrm{Cr}\left(\mathrm{H}_{2} \mathrm{O}\right)_{6}\right]^{3+}$ complex and besides ionic interactions we find a covalent contribution of ligand to metal charge transfer character. Based 
on our results we conclude that TFY spectra of 3d transition-metal ions and complexes in solution cannot be, in general, interpreted in terms of charge transfer to solvent effects.

\section{EXPERIMENTAL SECTION}

The samples consisted of aqueous solutions of $\mathrm{CrCl}_{3}$ with a concentration of $1 \mathrm{~mol} / \mathrm{l}$. The solutions were prepared at least 1 day before measurement to ensure known speciation of mostly hexacoordinated $\mathrm{Cr}^{3+}$. All spectra were measured at room temperature. Careful analysis of transmission-mode spectra of solution of various ages with supposedly different speciation showed not noticeable difference. Speciation can hence be neglected for the discussed effects. The experimental transmission-mode spectrum was measured on a thin film of the sample of approximately $1 \mu \mathrm{m}$ thickness sandwiched between two x-ray transparent windows in the set up described in ref. 42 at beamline PM3 at BESSYII. Both the TFY and the PFY spectra and the RIXS plane were measured on a liquid jet in vacuum (the detailed description of the set up will be published separately by Kunnus et al.) at beamlines U41 and U49 at BESSYII. The Cr RIXS plane and the Cr and O PFY spectra were measured with a Rowland-type RIXS spectrometer by detecting photons arising from fluorescence at the Cr L- and O K-edges under an angle of 90 degrees with respect to the propagation direction of the incident radiation. The polarization of the incident radiation was linear and parallel to the detection direction of the fluorescence (in the horizontal scattering plane). Polarization effects were shown to have minor influences (Kurian et al., unpublished results). TFY spectra were measured by detecting the total fluorescence from the sample with a GaAsP diode (Hamamatsu model G-112704). 


\section{THEORETICAL CALCULATIONS}

The simulations are based on the well-known formula for fluorescence-detected x-ray absorption spectroscopy and we used its implementation from Eisebitt et al. (31) which we modified to account for two fluorescing centers (unpublished results). This formula is used here to simulate all effects that one could call "x-ray optical effects" such as a variation of the relative absorption strength when the solute absorption edge is scanned, self absorption or saturation. We assume fluorescence to arise from two species only, $\mathrm{Cr}$ and O, labeled 1 and 2 respectively, and the TFY spectrum being the sum of the $\mathrm{Cr}\left(\mathrm{I}_{\mathrm{PFY} 1}\right)$ and $\mathrm{O}\left(\mathrm{I}_{\mathrm{PFY} 2}\right)$ PFY spectra:

$$
\begin{aligned}
I_{T F Y}\left(E_{\text {in }}\right) & =I_{P F Y 1}\left(E_{\text {in }}\right)+I_{P F Y 2}\left(E_{\text {in }}\right) \\
I_{P F Y 1}\left(E_{\text {in }}\right) & \propto \int \frac{f_{1}\left(E_{\text {in }}\right) \mu_{1}\left(E_{\text {in }}\right) \Phi_{1}\left(E_{\text {in }}, E_{\text {out } 1}\right)}{\mu_{1}\left(E_{\text {in }}\right)+\mu_{2}\left(E_{\text {in }}\right)+\mu_{b 1}\left(E_{\text {in }}\right)+\mu_{b 2}\left(E_{\text {in }}\right)+g\left[\mu_{1}\left(E_{\text {out } 1}\right)+\mu_{2}\left(E_{\text {out } 1}\right)+\mu_{b 1}\left(E_{\text {out } 1}\right)+\mu_{b 2}\left(E_{\text {out } 1}\right)\right]} d E_{\text {out } 1} \\
I_{P F Y 2}\left(E_{\text {in }}\right) & \propto \int \frac{f_{2}\left(E_{\text {in }}\right) \mu_{2}\left(E_{\text {in }}\right) \Phi_{2}\left(E_{\text {in }}, E_{\text {out } 2}\right)}{\mu_{1}\left(E_{\text {in }}\right)+\mu_{2}\left(E_{\text {in }}\right)+\mu_{b 1}\left(E_{\text {in }}\right)+\mu_{b 2}\left(E_{\text {in }}\right)+g\left[\mu_{1}\left(E_{\text {out } 2}\right)+\mu_{2}\left(E_{\text {out } 2}\right)+\mu_{b 1}\left(E_{\text {out } 2}\right)+\mu_{b 2}\left(E_{\text {out } 2}\right)\right]} d E_{\text {out } 2}
\end{aligned}
$$

with

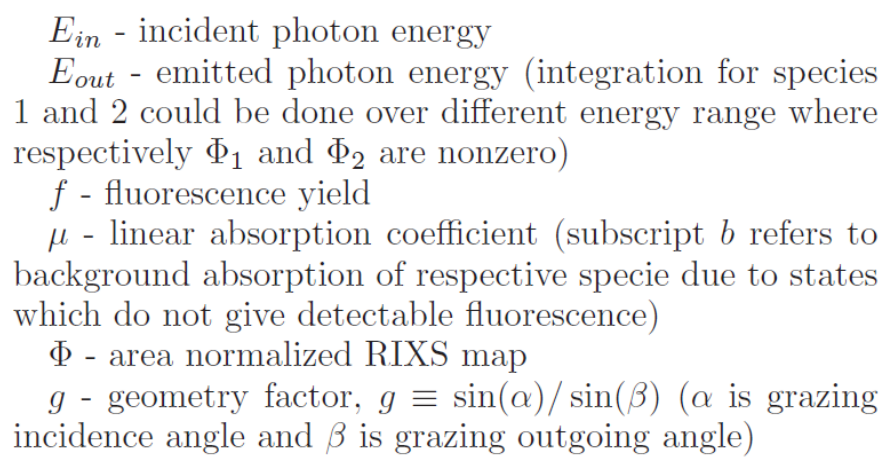

As experimental inputs for the simulations we used experimental x-ray absorption cross sections measured in transmission mode for the reported sample solution at the $\mathrm{Cr}$ L-edge $\left[\mu_{1}\left(\mathrm{E}_{\text {in }}\right)\right.$, shown in Figure $1(\mathrm{a})]$ and at the $\mathrm{O}$ K-edge $\left[\mu_{2}\left(\mathrm{E}_{\text {in }}\right)\right.$, not shown]. To describe the spectral distribution of 
the Cr L-edge emission, the experimental RIXS map shown in Figure 2(a) was used [ $\Phi_{1}\left(\mathrm{E}_{\mathrm{in}}, \mathrm{E}_{\text {out } 1}\right)$, RIXS spectra at different incident photon energies were normalized to unit area]. To describe the $\mathrm{O}$ K-emission an experimental off-resonance $\mathrm{x}$-ray emission spectrum (not shown) was used $\left[\Phi_{2}\left(\mathrm{E}_{\text {in }}, \mathrm{E}_{\text {out } 2}\right)\right]$. The samples thickness for the $\mathrm{O}$ K-edge transmission spectrum was well below $1 \mu \mathrm{m}$ in order to avoid saturation effects. For the geometry of the simulated TFY spectrum we assumed that the diode detects fluorescence under an angle of 45 degrees \pm 30 degrees corresponding approximately to the experimental implementation.

The restricted active space (RASSCF) calculations followed closely the scheme developed for $\mathrm{Ni}^{2+}$ (aq) (Josefsson et al., unpublished results). The explicit inclusion of static electron correlation through multi-configurational wave-functions is highly suitable for the calculation of L-edge x-ray spectra. Dynamical correlation was added in a perturbative treatment (RASPT2). A minimal active space of Cr2p(RAS1=3 containing at most one hole) and Cr3d(RAS2=5) was employed. Scalar relativistic RASPT2 calculations) for all resulting $2 \mathrm{p}^{6} 3 \mathrm{~d}^{3} / 2 \mathrm{p}^{5} 3 \mathrm{~d}^{4}$ (doublet, quartet) and $2 \mathrm{p}^{5} 3 \mathrm{~d}^{4}$ sextet states were performed on an optimized $\left[\mathrm{Cr}\left(\mathrm{H}_{2} \mathrm{O}\right)_{6}\right]^{3+}$ complex (CASPT2/TZVP: Cr-O=2.003 $\AA$ ). The spin-orbit matrix elements were computed in the stateinteraction framework over a basis of state-averaged RASSCF wavefunctions in $\mathrm{D}_{2 \mathrm{~h}}$ symmetry. The XA and RIXS spectra from the high-spin ground state were calculated with the resulting complex electronic states. Details will be published separately by Josefsson et al..

For the CFM calculations (39) we used $10 \mathrm{Dq}=2 \mathrm{eV}$ and a $20 \%$ reduction of the Slater integrals. The same broadening scheme was used for all calculations: $0.3 \mathrm{eV}$ and $0.6 \mathrm{eV}$ Lorentzian 
broadening at the $\mathrm{L}_{3}$ and $\mathrm{L}_{2}$ edges, respectively and $0.3 \mathrm{eV}$ Gaussian broadening (all values in FWHM) to account for the bandwidth of the incident radiation. 


\section{AUTHOR INFORMATION}

\section{Corresponding Author}

* E-mail: wernet@helmholtz-berlin.de, Phone+49 30 806213448, Fax: +49 30806212114.

\section{Author Contributions}

The manuscript was written through contributions of all authors. All authors have given approval to the final version of the manuscript.

\section{Funding Sources}

M.O. aknowledge support from the Swedish Research Council, Carl Tryggers Foundation, and Magnus Bergvall Foundation.

\section{ACKNOWLEDGMENTS}

We gratefully acknowledge support by the staff of the Helmholtz-Zentrum Berlin for support during the BESSYII beamtimes. We cordially thank Christian Weniger for support of the transmission measurements.

Supporting Information Available: Supporting text. This material is available free of charge via the Internet http://pubs.acs.org. 


\section{REFERENCES}

1 Stöhr, J. NEXAFS Spectroscopy, (Springer-Verlag, Berlin, 1992).

2 Khalil, M. , Marcus, M. A. , Smeigh, A. L. , McCusker, J. K. , Chong, H. H. W., Schoenlein, R. W. Picosecond Xray Absorption Spectroscopy of a Photoinduced Iron(II) Spin Crossover Reaction in Solution. J. Phys. Chem. A 2006, 110, 38-44.

3 Bressler, C., Milne, C., Pham, V.-T. , ElNahhas, A. , van der Veen, R. M. , Gawelda, W. , Johnson, S. , Beaud, P. , Grolimund, D. , Kaiser, M. , et al. Femtosecond XANES Study of the Light-Induced Spin Crossover Dynamics in an Iron(II) Complex. Science 2009, 323, 489-492.

4 Nozawa, S., Sato, T., Chollet, M., Ichiyanagi, K.,Tomita, A., Fujii, H., Adachi, S.-I., Koshihara, S.-Y. Direct Probing of Spin State Dynamics Coupled with Electronic and Structural Modifications by Picosecond TimeResolved XAFS J. Am. Chem. Soc. 2010, 132, 61-63.

5 Vankó, G., Glatzel, P., Pham, V.-T., Abela, R., Grolimund, D., Borca, C. N., Johnson, S. L., Milne, C. J., Bressler, C. Picosecond Time-Resolved X-Ray Emission Spectroscopy: Ultrafast Spin-State Determination in an Iron Complex. Angew. Chem. Int. Ed. 2010, 49, 5910-5912.

6 Huse, N., Cho, H., Hong, K., Jamula, L., de Groot, F. M., Kim, T. K., McCusker, J. K., Schoenlein, R. W. Femtosecond Soft X-ray Spectroscopy of Solvated Transition-Metal Complexes: Deciphering the Interplay of Electronic and Structural Dynamics. J. Phys. Chem. Lett. 2011, 2, 880-884.

7 Chen, L. X., Jager, W. J. H., Jennings, G., Gosztola, D. J., Munkholm, A., Hessler, J. P. Capturing a Photoexcited Molecular Structure Through Time-Domain X-ray Absorption Fine Structure. Science 2001, 292, 262-264.

8 Wernet, Ph., Gavrila, G., Godehusen, K., Weniger, C., Nibbering, E., Elsaesser, T., Eberhardt, W. Ultrafast Temperature Jump in Liquid Water Studied by a Novel Infrared Pump-x-ray Probe Technique. Appl. Phys. A 2008, 92, 511-516.

9 Huse, N., Wen, H., Nordlund, D., Szilagyi, E., Daranciang, D., Miller, T. A., Nilsson, A., Schoenlein, R. W., Lindenberg, A. M., Probing the Hydrogen-bond Network of Water via Time-resolved Soft X-ray Spectroscopy. Phys. Chem. Chem. Phys.2009, 11, 3951-3957.

10 Elles, C. G., Shkrob, I. A., Crowell, R. A., Arms, D. A., Landahl, E. C. Transient X-ray Absorption Spectroscopy of Hydrated Halogen Atom. J. Chem. Phys. 2008, 128, 061102:1-4.

11 Wernet, Ph. Electronic Structure in Real Time: Mapping Valence Electron Rearrangements During Chemical Reactions. Phys. Chem. Chem. Phys. 2011, 13, 16941-16954.

12 Björneholm, O., Nilsson, A., Sandell, A., Hernnäs, B., Martensson, N. Determination of Time Scales for ChargeTransfer Screening in Physisorbed Molecules. Phys. Rev. Lett. 1992, 68, 1892-1895.

13 Karis, O., Nilsson, A., Weinelt, M., Wiell, T., Puglia, C., Wassdahl, N., Mårtensson, N., Samant, M., Stöhr, J. One-Step and Two-Step Description of Deexcitation Processes in Weakly Interacting Systems. Phys. Rev. Lett. 1996, 76, 1380-1383.

14 Keller, C., Stichler, M., Comelli, G., Esch, F., Lizzit, S., Wurth, W., Menzel, D. Ultrafast Charge Transfer Times of Chemisorbed Species from Auger Resonant Raman Studies. Phys. Rev. Lett. 1998, 80, 1774-1777.

15 Wurth, W., Menzel, D. Ultrafast Electron Dynamics at Surfaces Probed by Resonant Auger Spectroscopy. Chem. Phys.2000, 251, 141-149. 
16 Schnadt, J., Brühwiler, P. A., Patthey, L., O'Shea, J. N., Södergren, S., Odelius, M., Ahuja, R., Karis, O., Bässler, M., Persson, P., et al. Experimental Evidence for Sub-3-fs Charge Transfer from an Aromatic Adsorbate to a Semiconductor. Nature 2002, 418, 620-623.

17 Brühwiler, P., Karis, O., Mårtensson, N. Charge-transfer Dynamics Studied Using Resonant Core Spectroscopies. Rev. Mod. Phys. 2002, 74, 703-740.

18 Föhlisch, A., Feulner, P., Hennies, F., Fink, A., Menzel, D., Sanchez-Portal, D., Echenique, P. M., Wurth, W. Direct Observation of Electron Dynamics in the Attosecond Domain. Nature 2005, 436, 373-376.

19 Nordlund, D., Ogasawara, H., Bluhm, H., Takahashi, O., Odelius, M., Nagasono, M., Pettersson, L. G. M., Nilsson, A. Probing the Electron Delocalization in Liquid Water and Ice at Attosecond Time Scales. Phys. Rev. Lett. 2007, 99, 217406:1-4.

20 Aziz, E. F., Ottosson, N., Faubel, M., Hertel, I. V., Winter, B. Interaction Between Liquid Water and Hydroxide Revealed by Core-hole De-excitation. Nature 2008, 455, 89-91.

21 Aziz, E. F., Rittmann-Frank, M. H., Lange, K. M., Bonhommeau, S., Chergui, M. Charge Transfer to Solvent Identified Using Dark Channel Fluorescence-yield L-edge Spectroscopy. Nat. Chem. 2010, 2, 853-857.

22 Bauer, M., Stalinski, T., Aziz, E. F. Insights into the Induced Ultrafast Electron Delocalization in Fe(CO) $)_{5}$ Using Dark Channel Fluorescence Yield X-ray Spectroscopy. Chem. Phys. Chem. 2011, 12, 2088-2091.

23 Lange, K., Kothe, A., Aziz, E. F. Chemistry in Solution: Recent Techniques and Applications Using Soft X-ray Spectroscopy. Phys. Chem. Chem. Phys. 2012, 14, 5331-5338.

24 Aziz, E. F., X-ray Spectroscopies Revealing the Structure and Dynamics of Metalloprotein Active Centers. J. Phys. Chem. Lett. 2011, 2, 320-326.

25 Nilsson, A., X-ray Spectroscopy: Transferring Electrons to Water. Nat. Chem. 2010, 2, 800-802.

26 Näslund, L.-Å., Cavalleri, M., Ogasawara, H., Wernet, Ph., Edwards, D. C., Sandstroem, M., Myneni, S., Nilsson, A., Pettersson, L. G. M. Direct Evidence of Orbital Mixing Between Water and Solvated Transition Metal Ions: An Oxygen 1s XAS and DFT Study of Aqueous Systems. J. Phys. Chem. A 2003, 107, 6869-6876.

27 Seidel, R., Ghadimi, S., Lange, K. M., Bonhommeau, S., Soldatov, M. A., Golnak, R., Kothe, A., Könnecke, R., Soldatov, A., Thürmer, S., et al. F. Origin of Dark-Channel X-ray Fluorescence from Transition-Metal Ions in Water. J. Am. Chem. Soc. 2012, 134, 1600-1605.

28 Soldatov, M. A., Lange, K. M., Gotz, M. D., Engel, N., Golnak, R., Kothe, A., Aziz, E. F. On the Origin of Dips in Total Fluorescence Yield X-ray Absorption Spectra: Partial and Inverse Partial Fluorescence Yield at the L-edge of Cobalt Aqueous Solution. Chem. Phys. Lett. 2012, 546, 164-167.

29 de Groot, F. M. F., Dips and Peaks in Fluorescence Yield X-ray Absorption Are Due To State-dependent Decay. Nat. Chem. 2012, 4, 766-767.

30 T. Z. Regier, A. J. Achkar, D. Peak, J. S. Tse, D. G. Hawthorn, Dark Channel Fluorescence Observations Result from Concentration Effects Rather than Solvent-solute Charge Transfer. Nat. Chem. 2012, 4, 765-766.

31 Eisebitt, S., Böske, T., Rubensson, J.-E., Eberhardt, W. Determination of Absorption Coefficients for Concentrated Samples by Fluorescence Detection, Phys. Rev. B 1993, 47, 14103-14109.

32 Achkar, A. J., Regier, T. Z., Wadati, H., Kim, Y.-J., Zhang, H., Hawthorn, D. G., Bulk Sensitive X-ray Absorption Spectroscopy Free of Self-absorption Effects. Phys. Rev. B 2011, 83, 081106(R):1-4. 
33 de Groot, F.M.F. Fluorescence Yield Detection: Why it Does Not Measure the X-ray Absorption Cross Section. Solid State Commun. 1994, 92, 991-995.

34 Krause, O. Atomic Radiative and Radiationless Yields for K and L Shells. J. Phys. Chem. Ref. Data 1979, 8, 307-328.

35 Coster, D., Kronig, R. De L. New Type of Auger Effect and its Influence on the X-ray Spectrum. Physica 1935, 2, 13-24.

36 Gotz, M. D., Soldatov, M. A., Lange, K. M., Engel, N., Golnak, R., Könnecke, R., Atak, K., Eberhardt, W., Aziz, E. F. Probing Coster-Kronig Transitions in Aqueous $\mathrm{Fe}^{2+}$ Solution Using Inverse Partial and Partial Fluorescence Yield at the L-Edge. J. Phys. Chem. Lett. 2012, 3, 1619-1623.

37 Aziz, E. F., Ottosson, N., Bonhommeau, S., Bergmann, N., Eberhardt, W., Chergui, M. Probing the Electronic Structure of the Hemoglobin Active Center in Physiological Solutions. Phys. Rev. Lett. 2009, 102, 068103:1-4.

38 Bergmann, N., Bonhommeau, S., Lange, K. M., Greil, S. M., Eisebitt, S., de Groot, F.M.F, Chergui, M., Aziz, E. F. On the Enzymatic Activity of Catalase: An Iron L-edge X-ray Absorption Study of the Active Centre. Phys. Chem. Chem. Phys.2010, 12, 4827-4832.

39 Stavitski, E., de Groot, F.M.F., The CTM4XAS Program for EELS and XAS Spectral Shape Analysis of Transition Metal L edges. Micron 2010, 41, 687-694.

40 E. F. Aziz, K. M. Lange, S. Bonhommeau, M. Chergui, Reply to 'Dark channel fluorescence...' and 'Dips and peaks...'. Nat. Chem. 2012, 4, 767-768.

41 K.M. Lange, E. Suljoti, E.F. Aziz, Resonant Inelastic X-Ray Scattering as a Probe of Molecular Structure and Electron Dynamics in Solutions, Journal of Electron Spectroscopy and Related Phenomena 2012, doi:10.1016/j.elspec.2012.09.010

42 Schreck, S., Gavrila, G., Weniger, C., Wernet, Ph. A Sample Holder for Soft X-ray Absorption Spectroscopy of Liquids in Transmission Mode. Rev. Sci. Instrum. 2011, 82, 103101:1-10. 